\title{
Radiofrequency Catheter Ablation of Left-sided Accessory Atrioventricular Pathways at Atrial Insertion Sites
}

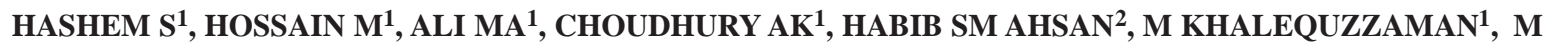 \\ AKHTARUZZAMAN $^{1}$, S NABI ${ }^{1}$, TA CHOUDHURY ${ }^{1}$, N I SHARAFAT ${ }^{1}$ \\ ${ }^{1}$ Dept. of Cardiology, National Institute of Cardiovascular Disease (NICVD), Dhaka, ${ }^{2}$ Dept. of Cardiology, Bangabandhu Sheikh \\ Mujib Medical University (BSMMU), Dhaka \\ Address of Correspondence: Dr Sabina Hashem, Department of Cardiology, \\ National Institute of Cardiovascular Diseases, Dhaka,
}

\begin{abstract}
:
Background: This study assessed the efficacy and safety of techniques used to ablate left-sided accessory atrioventricular pathways at atrial insertion sites by retrograde aortic approach. Radiofrequency catheter ablation of left-sided accessory pathways by way of retrograde aortic approach can be highly successful.

Methods: This study were done in the department of Cardiology, National Institute of Cardiovascular Diseases (NICVD), Dhaka from June 2009 and March 2010, a total 30 patients with recurrent SVT who has left sided accessory atrioventricular pathways found after electrophysiological study, underwent attempted radiofrequency catheter ablation of one or more accessory atrioventricular pathways by retrograde aortic approach. The ablation catheter was inserted into the femoral artery and passed retrogradely across the aortic valve. Once an optimal target site has been identified, radiofrequency energy at a pre-selected temperature of $55-60^{\circ} \mathrm{C}$ and power output set at 50 watts was delivered through the ablation catheter. Loss of preexcitation or interruption of the tachycardia within 5 seconds of RF application was considered effective and RF current was continued in this location 30 to 60 seconds. Ablation success was defined at completion of procedure as acutely successful or unsuccessful on the basis of successful elimination of all ablation targets.

Results: During EP study it was found that 9 patients had concealed accessory pathways and 21 had manifest pre-excitation. WPW left-lateral pathway was present in 10 (33.3\%), concealed left lateral in 08 (26.7\%), WPW left posterior in 11 (36.7\%), concealed left posterior in 1 (3.3\%). Out of 30 accessory pathways, 24 were successfully interrupted with radiofrequency catheter ablation with a primary success rate of $80 \%$. The ratio of atrial/ventricular electrogram in successful sites was $0.83 \pm 0.27$ (0.53-1.46). There were no major complications with retrograde aortic approach. Six failed patients were ablated via transseptal method.

Conclusion: The retrograde atrial insertion approach to left-sided accessory pathway ablation is very safe and high effective, especially suitable for the failed patients by using retrograde ventricular insertion ablation procedure and by using single-catheter ablation of accessory pathway.
\end{abstract}

Key Word: Tachycardia, supraventricular, accessory pathway, retrograde aortic approach, atrial insertion site, catheter ablation

\section{Introduction:}

The technique of radiofrequency catheter ablation has been widely applied to cure the patients with symptomatic supraventricular tachycardia (SVT) involving accessory atrioventricular pathways. Most investigators used the methods for ablating left-sided accessory pathway by inserting ablation catheter through the femoral artery and advancing it retrograde across the aortic valve and positioning it below the mitral annulus. ${ }^{1-3}$ Several electrophysiologist made access to the atrial aspect of the mitral annulus obtained by way of transseptal catheterization. $^{4-6}$ Since its introduction in 1986 retrograde approach has been the most widely used techniques for ablation of left sided accessory pathways. Most ablation procedures reported were carried out from the ventricular aspect by inserting the ablation catheter through the femoral artery and advancing it retrograde across the aortic valve. Advantages include ready access via the femoral artery, familiarity by most electro physiologists, no need for specialized sheaths or equipments and feasibility following full anticoagulation ${ }^{7}$. This study investigated the efficacy and safety of catheter ablation left-sided accessory pathways by positioning ablation catheter above the mitral annulus by way of retrograde aortic approach. Although the retrograde aortic approach has inherent risks related to arterial access, across the aortic valve and catheter manipulation in the left ventricle, it has achieved a high success rate. However, for accessory pathways with an epicardial location, with an oblique course or most interiorly located, ablation on 
the atrial aspect of mitral annulus by transseptal approach has achieved many advantages. Alternatively, reports have described access to the atrial aspect of the mitral annulus obtained by way of transseptal catheterization, which may avoid potential problems associated with the retrograde approach. ${ }^{8-10}$

\section{Methods:}

From June 2009 and March 2010, a total 30 patients with recurrent SVT who has left sided accessory atrioventricular pathways found after electrophysiological study, underwent attempted radiofrequency catheter ablation of one or more accessory atrioventricular pathways in the department of Cardiology, National Institute of Cardiovascular Diseases (NICVD), Dhaka. These patients experienced catheter ablation of accessory pathways atrial insertion sites by way of retrograde aortic approach. Patient excluded from the study were; symptomatic coronary artery disease, complex congenital cardiac anomaly, repaired inter atrial communication, peripheral arterial disease or aortic disease, mechanical prosthesis in the aortic or mitral valve position and prior catheter ablation.

Electrophysiology study: After informed written consent, initial evaluation of the patient was done by history, clinical examination and relevant investigations. The procedure was performed after an 8-hour fasting, after interruption of antiarrhythmic drugs for five half-lives and of amiodarone hydrochloride for a month. Under sedation with intravenous midazolam electrophysiologic studies were performed. Four 7F catheters was introduced through punctures in the right femoral vein and placed in high right atrium, bundle of His region, coronary sinus and right ventricle guided by fluoroscopy in order to record intracardiac electrogram and do electrophisiology test. Preliminary mapping was performed with a quadripolar catheter in the coronary sinus proceeding to more precise mapping with the ablation catheter. After diagnostic confirmation by electrophysiological study (EPS), the patients were assigned the retrograde aortic method . As part of the routine, intravenous heparin (5000 IU bolus IV followed by 1000 IU per hour)was used for anticoagulation.

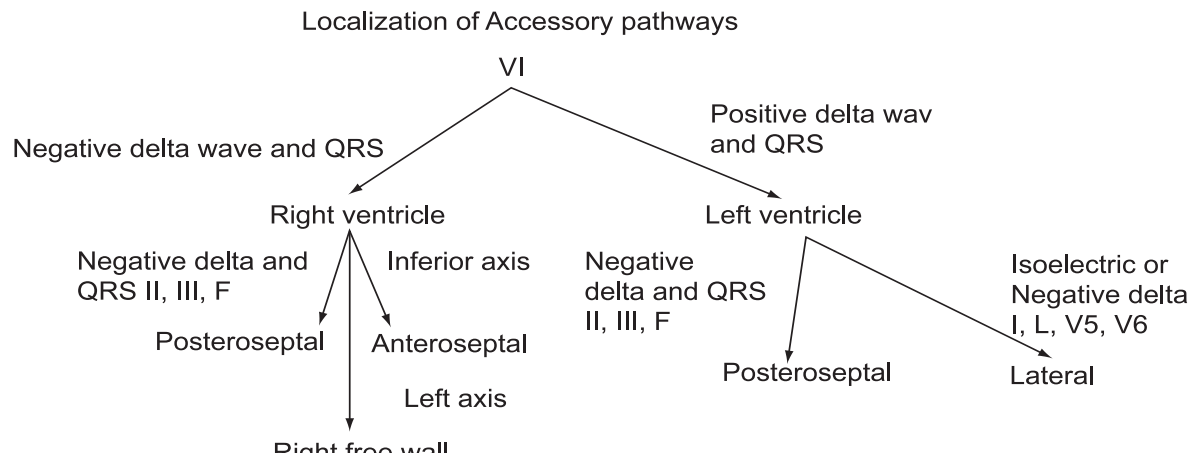

Right free wall
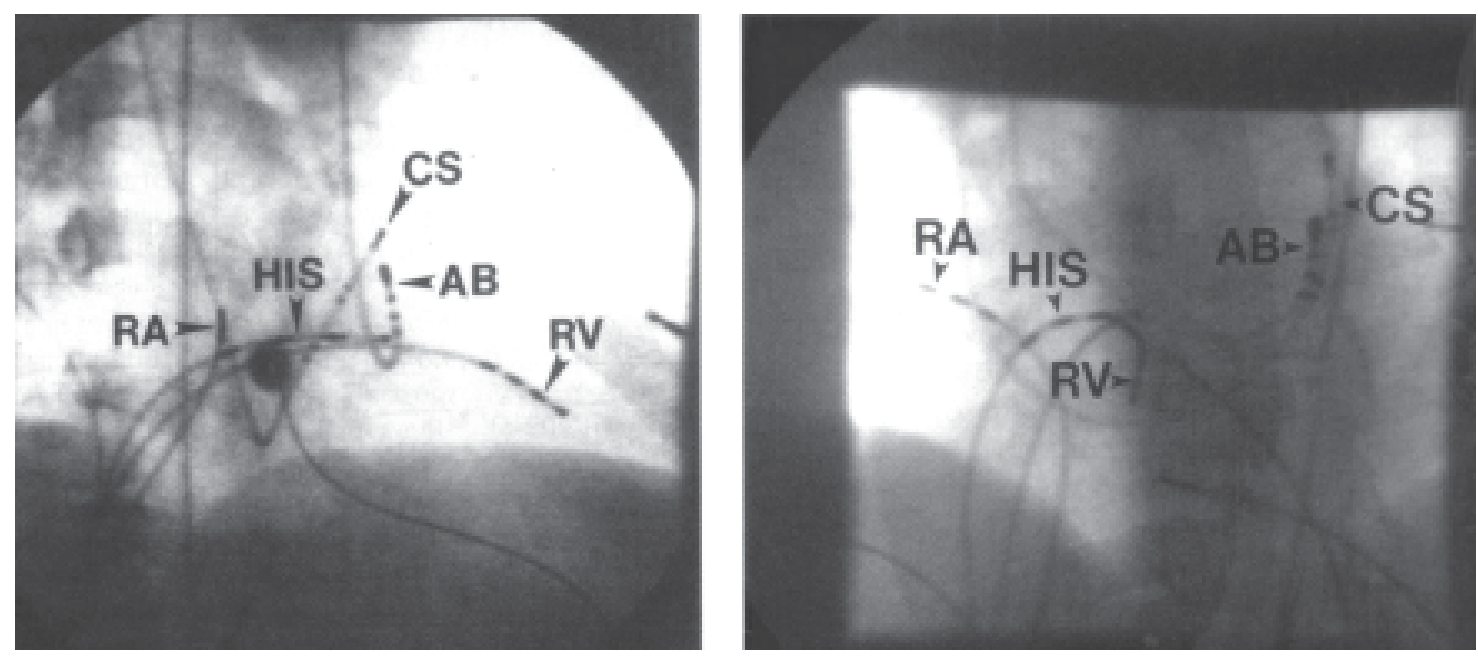

Fig.-1: Catheter positions in right anterior oblique (A) and left anterior oblique (B) for ablation of left free wall accessory pathway by the retrograde transaortic approach. 
Ablation protocol: After preliminary localization of the accessory pathway, precise mapping within the targeted region was performed with the ablation catheter, which was a 7Fquadripolar electrode catheter with a 4-mm distal electrode an inter electrode spacing of 2 or $5 \mathrm{~mm}$ and a deflectable curve

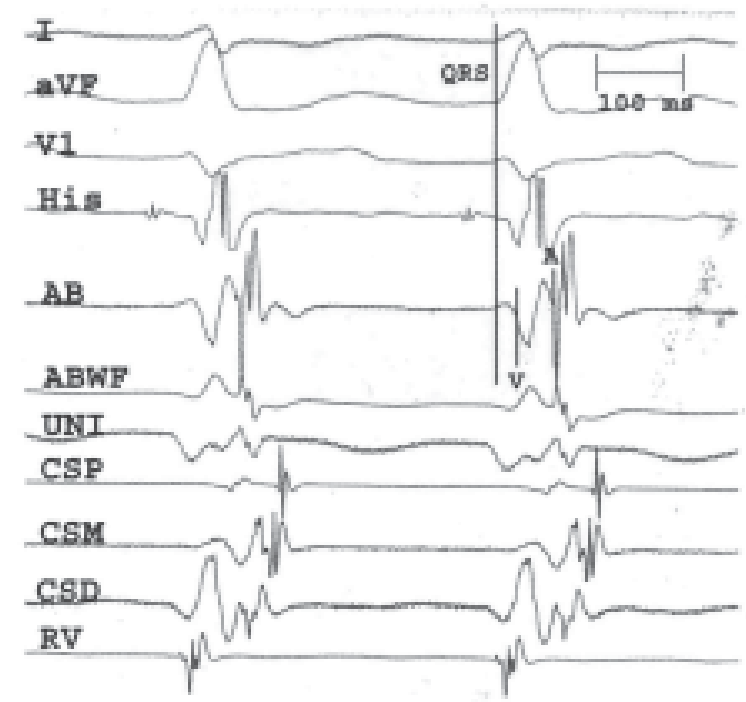

Fig.-2: Electrograms from successful ablation site during mapping of retrograde accessory pathway (AP) conduction in orthodromic reciprocating tachycardia. The distal coronary sinus is earliest on this catheter. The QRS onset (QRS) to local atrial electrogram (A) interval on the ablation catheter is $68 \mathrm{msec}$ and is shown by the vertical lines. The local ventricular $(V)$ to atrial interval on the ablation electrodes is 40 msec. Note the isoelectric morphology of the atrial component of the electrogram on the wide filtered ablation electrogram ( $A B W F$ ) representing the atrial insertion point of the AP.

The ablation catheter was inserted into the femoral artery and passed retrogradely across the aortic valve into the left ventricle and the tip deflected to target sites under the mitral valve as close as possible to the annulus. Initial target sites were chosen on the basis of mapping within the coronary sinus. The ablation catheter was then manipulated to a site that had the shortest interval between the local atrial and ventricular electrograms and the earliest ventricular activation during sinus rhythm or atrial pacing in the case of manifest pre-excitation and the site of the earliest retrograde atrial activation during orthodromic reciprocating tachycardia or ventricular pacing. Once an optimal target site has been identified, radiofrequency energy at a pre-selected temperature of $55-60{ }^{0} \mathrm{C}$ and power output set at 50 watts was delivered through the ablation catheter. Loss of preexcitation or interruption of the tachycardia within 5 seconds of RF application was considered effective and RF current was continued in this location 30 to 60 seconds. If several applications of radiofrequency energy failed to abolish anterograde and retrograde conduction over the accessory pathway, the catheter tip was fully straightened and rotated in a counterclockwise fashion to reach the inflow tract where, by deflecting the tip, the catheter was advanced into the left atrium. The catheter was then withdrawn and rotated clockwise or Counter clockwise to reach the anterior and posterior annulus areas respectively. After target site was repeatedly confirmed, radiofrequency energy was delivered .

Procedural Success: Ablation success was defined at completion of procedure as acutely successful or unsuccessful on the basis of successful elimination of all ablation targets. Accessory pathways ablation was considered successful if both antegrade and retrograde conduction through the pathway was abolished (in overt pathways), if retrograde conduction was abolished in concealed pathways or if antegrade pathway conduction was abolished in pathways with antegrade conduction only.

Follow up: After the ablation the catheters and sheaths were removed and hemostasis was achieved by manual compression. Patients were transferred to coronary care unit for monitoring. A 12 lead ECG \& Echocardiographic evaluation was performed on the second post ablation day in hospital and patients were discharged home.

\section{Results:}

A total 30 patient for radio frequency ablation of left free wall accessory pathway where selected for retrograde aortic approach. The mean age of the patients was $37.63 \pm 10.49$ years( 22 were male and 8 were female). Nine patients had concealed accessory pathways, and 21 had manifest preexcitation. Eleven (36.7\%) patient had SVT with WPW and 8 (26.7\%) had SVT without WPW, 11(36.7\%) atrial fibrillation with WPW. All patients with SVT experienced symptomatic atrioventricular reentrant tachycardia for an average of 5-6 years, undergone unsuccessful medical therapy regimens before being referred for ablation.

Table1: showed that mean age of study population $63 \pm$ 10.49 years. Among them 22(73.3\%) were male and $8(26.7 \%)$ were female .Study also revealed that $13 \%$ patient were diabetic, $23.3 \%$ patient were hypertensive, $6.7 \%$ patient had bronchial asthma, and $6.7 \%$ patient had recurrent epilepsy. All patient took antiarrahythmic drugs before ablation. Among them 18 patient took verapamil, 3 patient took Metoprolol, 6 patients took amiodarone, 3 patient Sotalol. 2 patient was suffering from Valvular heart disease and 1 patient form atrial septal defect . 
Table-I

Baseline characteristics of patients

\begin{tabular}{lc}
\hline Age (in year) & \\
Mean \pm SD & $37.63 \pm 10.44$ \\
Sex & $n(\%)$ \\
- Male & $22(73.3)$ \\
- $\quad$ Female & $8(26.7)$ \\
Concomitant Medical illlness & $\mathrm{n}(\%)$ \\
- $\quad$ DM & $4(13.3)$ \\
- HTN & $7(23.3)$ \\
- $\quad$ Bronchial asthma & $2(6.7)$ \\
- $\quad$ Epilepsy & $2(6.7)$ \\
Antiarrhythmic Drugs used before ablation & $\mathrm{n}(\%)$ \\
- $\quad$ Verapamil & $18(60.0)$ \\
- $\quad$ Metoprolol & $3(7.3)$ \\
- Amiodarone & $6(20.0)$ \\
- Sotalol & $3(10.0)$ \\
Structural Heart Disease & $\mathrm{n}(\%)$ \\
- $\quad$ Valvular heart disease & $2(6.7)$ \\
- $\quad$ Atrial Septal defect & $1(3.3)$ \\
\hline
\end{tabular}

\#Figure within parentheses indicates in percentage.

Table 2 shows the locations of pathways in the left free wall. 21 accessory pathways were manifest and 09 were concealed. WPW left-lateral pathway was present in 10 (33.3\%) , concealed left lateral in 08 (26.7\%),WPW left posterior in $11(36.7 \%)$, concealed left posterior in 1 (3.3\%). This table revealed that manifest and left lateral pathway is commonest.

Table-II

Location of different pathways

\begin{tabular}{lc}
\hline Manifest (WPW) & Total-21 \\
\hline Left lateral & $10(33.3)$ \\
Left posterior & $11(36.7)$ \\
Left anterolateral & $0(0)$ \\
Concealed & Total-09 \\
left lateral & $08(26.7)$ \\
left posterior & $1(3.3)$ \\
\hline
\end{tabular}

Table-III shows electrophysiological characteristics. Sinus Cycle length was 595 \pm 65 millisecond, AH interval $85 \pm$ 20 millisecond, HV interval $42.8 \pm 6.2$ millisecond and

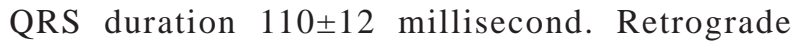
Wenckebach cycle were $220 \pm 42$ millisecond, Antegrade Wenckebach cycle were $330 \pm 79$ millisecond, Accessory pathway BCL 340 \pm 50 millisecond, Accessory pathway ERP 360 \pm 40 millisecond and SVT Cycle length 329 \pm 46 millisecond.
Table-III

Electro physiological characteristics

\begin{tabular}{lc}
\hline Parameters & Duration in ms \\
\hline Sinus Cycle length & $595 \pm 65$ \\
AH interval & $85 \pm 20$ \\
HV interval & $42.8 \pm 6.2$ \\
QRS interval & $110 \pm 12$ \\
Retrograde Wenckebach cycle & $220 \pm 42$ \\
Antegrade Wenckebach cycle & $330 \pm 79$ \\
Accessory pathway BCL & $340 \pm 50$ \\
Accessory pathway ERP & $360 \pm 40$ \\
SVT Cycle length & $329 \pm 46$ \\
\hline
\end{tabular}

Values are presented as mean \pm SD. SVT- supra ventricular tachycardia. AH- atrial potential to His potential. HV- His potential to ventricular potential interval, Antegrade and retrograde wenckeback cycle. SVT cycle length. Accessory pathway BCL -Accessory pathway block cycle length. Accessory pathway ERP-Accessory pathway effective refractory period.

Table-IV showed puncture time was $8.10 \pm 2.17$ minutes, ablation time was $39.73 \pm 15.55$ and number of radio frequency application $3.17 \pm 1.90$. The total procedural time $119.40 \pm 42.28$ and fluoroscopy time was $20.43 \pm$ 8.09

Table-IV

Parameters of radiofrequency application

\begin{tabular}{lc}
\hline Parameters & Time in min (mean \pm SD) \\
\hline Puncture time & $8.10 \pm 2.17$ \\
Ablation time & $39.73 \pm 15.55$ \\
Fluoroscopy time & $20.43 \pm 8.09$ \\
Total time of procedure & $119.40 \pm 42.28$ \\
No.of radiofrequency application & $3.17 \pm 1.90$ \\
\hline
\end{tabular}

"Figure within parentheses indicates in percentage.

Table-V showed that among 30 patient radiofrequency ablation was successful in 24 patients with $80 \%$ success rate.

Table-V

Distribution of success rates for ablation

\begin{tabular}{lc}
\hline Parameters & Percentage \\
\hline Successful & $24(80)^{\#}$ \\
Unsuccessful & $6(20)$ \\
\hline Total & $30(100.0)$ \\
\hline
\end{tabular}

\#Figure within parentheses indicates in percentage 
Table-VI There were no major complications but some minor complications observed. Haematoma was observed only in $3(10 \%)$ patient, Other complications include hypotension 2(6.7\%) and vasovagal reaction 1 (3.3\%).

\section{Table-VI}

Distribution of complications

\begin{tabular}{lc}
\hline Patterns of complications & Frequency in \% \\
\hline - Haemorrhage & $0(0)$ \\
- Haematoma & $3(10.0)$ \\
- Hypotension & $2(6.7)$ \\
- & Vasovagal reaction \\
\hline
\end{tabular}

${ }^{\#}$ Figure within parentheses indicates in percentage.

\section{Discussion:}

Among two recomended approaches for radiofrequency ablation of left free wall accessory pathways at atrial insertion site namely retrograde aortic \& transseptal (TS) approach, retrograde aortic approach was known to be the conventional method. ${ }^{11}$ Several investigators had reported large clinical series of accessory atrioventricular pathway ventricular insertion radiofrequency ablation. ${ }^{1-3}$ High rates of success using ventricular insertion ablation was reported. Other reports had described access to the atrial inserted sites by transseptal approach and same rates of success using atrial insertion ablation was achieved ${ }^{4-6 .}$ This prospective study was done to observe safety and efficacy of retrograde aortic approach at atrial insersion site in 30 cases. The study was done in NICVD from June 2009 and March 2010 and mean age of patient was37.63 \pm 10.49 . Similar age incident reported by silva et $\mathrm{al}^{12}$ and lash et al ${ }^{13}$ mean age were $34.3 \pm 11$ years. Among them 22 were male and 8 were female and showed male preponderance. Silva et al ${ }^{12}$ observed among 100 patients, 56 patients were male and also demonstrated male preponderance. Among the study population eleven (36.7\%) patient had SVT with WPW and 8 (26.7\%) had SVT without WPW, 11(36.7\%) atrial fibrillation with WPW. All patients with SVT experienced symptomatic atrioventricular reentrant tachycardia for an average of 56 years, undergone unsuccessful medical therapy regimens before being referred for ablation. During EP study it was found that nine patients had concealed accessory pathways, and 21 had manifest pre-excitation and WPW left-lateral pathway was present in 10 (33.3\%), concealed left lateral in 08 (26.7\%), WPW left posterior in 11 (36.7\%), concealed left posterior in 1 (3.3\%). Regarding the location of accessory pathways manifest pathways was found to be $70 \%$ and left lateral (73\% ) which prevailed, similar results reported by couple of studies by Silva et al. ${ }^{12}$ From the experience of catheter ablation of left sided accessory pathway in 30 patients suggested that the indication of mapping and ablation atrial insertion sites by way of trans aortic approach was as follows: First, the ablation catheter advanced easily into the left atrium by the retrograde aortic approach, and radiofrequency current was tried to discharge on top of the mitral annulus in order to decrease fluoroscopy time.

Second, it was difficult to record fine atrial wave atrioventricular electrogram from the ventricular aspect of the mitral annulus, or failed to abolish accessory pathway after several tried ablations. Third, in rare left antero-septal accessory pathway, operators could not manipulate the ablation catheter tip under the mitral annulus in left ventricle.

Fourth, when electrode catheter could not be inserted into coronary sinus, single-catheter ablation of accessory pathway was performed. If ablation catheter was manipulated within the left atrium, the catheter was easily rotated clockwise or counterclockwise for mapping and ablating accessory pathways atrial insertion sites. Swartz $\mathrm{el} \mathrm{al}^{4}$ thinks that in some patients, there relatively large amplitude and long duration local ventricular electrogram can be recorded during retrograde atrial insertion mapping, and overlap and obscure low-amplitude accessory pathway activity. In the present study, when the ablation catheter was advanced the left atrium, and recorded large amplitude of atrial electrograms and small amplitude of ventricular electrograms, the catheter should be slowly withdrawn and recorded an atrial electrogram-to-ventricular electrogram amplitude ratio of 1.2 to 1.5 in sinus rhythm. At that time, the catheter was rotated clockwise or counterclockwise to map because the catheter may best ability. If the catheter recorded very short local electrogram atrio-ventricular or ventriculo atrial interval, the catheter withdrew slowly and atrial electrogram-to ventricular electrogram amplitude ratio was less than1.0, radiofrequency energy was tried to discharge to eliminate accessory pathway conduction. But, at that time, the ablation catheter might be easy to slip back into the ventricle, especially during orthodromic reciprocating tachycardia or ventricular pacing. Therefore, it was preferred to ablate of manifest accessory pathway during sinus rhythm and concealed accessory pathway during tachycardia or ventricular pacing. Otherwise, the mapping and ablation of left anteroseptal or left posteroseptal accessory pathways atrial insertions might be difficult. The study revealed ablation of left antero septal accessory pathway atrial insertion might be 
performed the same way of transseptal approach as by way of retrograde aortic approach and the ablation of left postero septal accessory pathway should be performed_in ventricular insertion by way of retrograde aortic approach. This study showed radiofrequency ablation was successfully done in 24 patients with $80 \%$ success rate. But initial failure of retrograde approach in 6 patients successful ablation was done by transseptal approach in separate session. Crossover to transsptal method was done during a separate session to avoid complication related to access, with a total efficacy of $100 \%$. Couple of studies reported a similar success rate. ${ }^{13.15,16}$ They also compared the efficacy and safety of transseptal and retrograde approach. Silva et al ${ }^{15}$ demonstrated success rate of (100\% versus 99\% ), Lesh et al ${ }^{13}$ ( $92 \%$ versus $85 \%$ ), Montenero et $\mathrm{al}^{16}$ ( $96 \%$ versus $88 \%$ ) in transseptal and retrograde approach respectively. All the previous study showed almost $100 \%$ success after crossover. De Ponti et, ${ }^{11}$ Sorbera et $\mathrm{al}^{24}$ in separate studies reported higher success rates for patient treated with transeptal method .Saul et $\mathrm{al}^{25}$ in a retrospective study using both the techniques found overall success rate of $100 \%$ for ablation of left free wall accessory pathways. Although it may be reached the Accessory pathways at atrial insertion using both techniques with high efficacy, ${ }^{6,8}$ the mapping of the Accessory pathways at ventricular insertion using the RAA technique was preferred since this approach provides greater stability to the catheter. In a subjective analysis, the operators found more difficulty in positioning the catheter via RAA in the anterolateral pathways. Overall the studies suggest that the differences found between the techniques may be related to the learning curve and to the experience of the operator, rather than to the specific technique used. ${ }^{14,15}$ When this study began ,the group was more experienced with procedures via RA. These results may be more expressive if a new study was carried out in the present, since the team now has a similar amount of experience with both approaches. As other authors reported $^{16-18}$ ablation of left free wall accessory pathway from the ventricular aspect of mitral annulus, failures occurred in 14 patients for an ideal local electrogram could not be recorded or accessory pathway could not be blocked permanently even with an ideal local electrogram. But, all of whom were successfully treated by ablation the atrial insertion site in a separate session - transseptal approach. In fact, unsuccessful radiofrequency delivery at a site with ideal local electrogram is uncommon, this may be contributed to a large lesion diameter by ablation, or ablation not only from the ventricular aspect but also the atrial aspect. There were no major complications such as femoral artery pseudo aneurysms, femoral arteriovenus fistula or aortic valve damage etc related to retrograde approach. Minor complications found in 6 patients , most of the complications were related to vascular access site haematoma and bleeding, which resolved spontaneously, leaving no sequelae. With retrograde aortic procedures, complications includes femoral hematomas, thrombotic iliac artery occlusion femoral artery pseudoaneurysms, femoral arterovenous fistula, aortic valve damage, coronary artery thrombosis, inadvertent coronary artery cannulation ${ }^{19,20}$ may be developed. Whereas, ablation leftsided accessory pathways by transseptal approach, the inherent risks or deficiency of arterial catheter manipulation and retrograde left ventricular catheterization in ablation of the ventricular insertion are avoided. Perforation of the aorta, cardiac tamponade and death associated with interatrial septum puncture have been reported in hemodynamic evaluation of PTMV. ${ }^{21,22}$ The most common are vascular complications in connection with the arterial puncture and pericardial effusion resulting from the puncture of the atrial wall during the TS puncture. ${ }^{23}$ Minich et all ${ }^{20}$ detected $30 \%$ new mild aortic regurgitation cases after procedures via RA. However, the clinical significance of these findings has not yet been determined. Greater complications were described by Calkins et $\mathrm{al}^{3}$ in 6 out of 158 patients submitted to the RA technique, including vascular complications, coronary thrombosis and aortic valve perforation Lesh et all, ${ }^{10}$ reported a case of dissection of the left coronary artery during the RA technique followed by myocardial infarction. Conversely, the potential of cardiac perforation during transseptal catheterization may occur in transseptal approach.

\section{Conclusion:}

In our experience, retrograde aortic approach is a valuable tool for left heart catheterization, such as in radiofrequency catheter ablation of a left-sided arrhythmogenic substrate. Effort was made to simplify the procedure without compromising safety, Nevertheless, it is a demanding procedure: it requires a particular expertise and should not be carried out by non-specialist physicians in centers in which the yearly number of patients undergoing the procedure could be limited. Moreover, particular care must be taken in manipulating the catheters throughout the procedure. No clinically relevant major complication related to catheterization procedure itself occurred in this study. Radiofrequency catheter ablation of different cardiac arrhythmias by the retrograde aortic approach achieves an excellent success rate. Finally ablation of the 
atrial insertion site of accessory pathways by the retrograde aortic approach is very safe and high effective, especially suitable for the failed patients by using retrograde ventricular insertion ablation procedure and by using single-catheter ablation.

\section{References:}

1. Calkins H, Langberg J, Sousa J, et al. Radiofrequency catheter ablation of accessory atrioventricular connections 5 in 250 patients. Abbreviated therapeutic approach to Wolff-Parkinson-White syndrome. Circulation 1992;85:1337.

2. Jackman WM, Wang X, Friday KJ, et al. Catheter ablationof accessory atrioventricular pathways ( Wolff-Parkinson- 6.White syndrome) by radiofrequency current. N Engl J Med1991, 324:1605.

3. Chen S A, Tsang WP, Hsia CP, et al. Radiofrequency catheterablation for treatment of Wolff-Parkinson-White syndrome short-and long-term follow-up. International J Cardiol 1992;37:199.

4. Swartz JF, Tracy CM, Fletcher RD. Radiofrequency endocardial catheter ablation of accessory atrioventricular pathway atrial insertion sites. Circulation 1993; 87:487.

5. Lesh MD, Van Hare GF, Scheinman MM, et al. Comparison of the retrograde and transseptal methods for ablation of left free wall accessory pathways. J Am Coll Cardiol 1993; 22:542.

6. Saul JP, Hulse JE, Wang D, et al. Catheter ablation of accessory atrioventricular pathways in young patients: Use of long vascular sheaths, the transseptal approach and a retrograde left posterior parallel approach. J Am Coll Cardiol 1993; 21:571

7. Wood MA. Ablation of Free wall accessory pathways. In catheter ablation of cardiac arrhythmias. eds. Huang SKS and Wood MA. $1^{\text {st }}$ edn. 2006. Sauders Elsevier. Philadelphia.: 371-98.

8. Deshpande SS, Bremner S, Sra JS, et al. Ablation of left freewall accessory pathway using radiofrequency energy at the atrial insertion site: transseptal versus transaortic approach. $J$ Cardiovasc Electrophysiol 1994; 5:219 .

9. Changsheng MA, Dong J,Yang X et al. Transseptal ApproachAn indispensable complement to retrograde aortic approach for Radiofrequency catheter ablation of left sided accessory pathways. J HK Coll Cardiol 1995; 3.

10. Lesh MD, Van Hare GF, Scheinman MM, Ports TA, Epstein LA. Comparison of the retrograde and transseptal methods for ablation of left free wall accessory pathways. J Am Coll Cardiol. 1993; 22:542-49.
11. Changsheng Ma, Jinrong Zhang. The experience of 200 cases of percutaneous transluminal mitral valvuloplasty. ChineseJ Interventional Cardiol 1993; 1:28-30.

12. Marcio Augusto Silva, Elenir Nadalin, Alessandro Kraemmer, Radiofrequency ablation of left accessory pathways by transseptal approach. Arq. Bras,Cardiol 2006; 86(5) :1-9.

13. Lesh Md, Van Hare GF, Scheinman MM, et al. Comparison of retrograde and transseptal methods for ablation of left free wall accessory pathways. J Am Coll Cardiol 1993; 22:532-9.

14. Vora AM, McMahon S, Jazayeri MR, Dhala AA. Ablation of atrial insertion sites of left-sided accessory pathways in children: efficacy and safety of transseptal versus transaortic approach. Pediatr Cardiol 1997; 18: 332-8.

15. Law IH, Eischbach PS, Leroy S, Lloyd TR, Rocchini AP, Dick M 1 Ideas to left atrium for delivery of radiofrequency ablation in young patients: retrograde aortic vs transseptal approach. Pediatr Cardiol 2001; 22: 204-9.

16. Langberg JJ, Man KC, Vorperian VR, et al. Recognition and catheter ablation of subepicardial accessory pathways. J AmColl Cardiol 1993; 22:1100-4.

17. Wang X, McClelland J, Beckman K, et al. Left free wall accessory pathways which require ablation from the coronary sinus: unique coronary sinus electrogram pattern (abstract).Circulation 1992;86(suppl I):I-81.

18. Haissaguerre M, Gaita F, Fischer B, et al. Radio-frequency catheter ablation of left lateral accessory pathways via the coronary sinus. Circulation 1992; 86:1464-8.

19. Calkins H, Langberg J, Sousa J, et al. Radiofrequency catheter ablation of accessory atrioventricular connections Abbreviated therapeutic approach to Wolff-Parkinson White syndrome. Circulation 1992; 85:1337-46.

20. Minichi, Snider A, Dick M II. Doppler detection of valvular regurgitation after radiofrequency ablation of accessory connections. Am J Cardiol 1992; 70:116-8. 11.

21. Mullins CE. Transseptal left heart catheterization: Experience with a new technique in pediatric and adult patients. Pediatric Cardiol 1983; 4:239-246.

22. Libanoff A, Silver A. Complications of transseptal left heart catheterization. Am J Cardiol 1956; 16:390-3.

23. Manolis AS, Wang PJ, Mark Estes NA, Dhala AA, Blanck Zl, Bajwa TK, et al. Radiofrequency ablation of left-sided accessory pathways: trans aortic versus transseptal approach. Am Heart J. 1994; 128: 896-902. 